\title{
Urban Smart Growth Mathematical Model and Application
}

\author{
Geng Liu ${ }^{1, ~ *, ~ X i a o ~} \mathrm{Han}^{2}$, Zhen $\mathrm{Li}^{3}$ \\ ${ }^{1}$ Basic Teaching Department, Rongcheng College of Harbin University of Science and Technology, Weihai, P. R. China \\ ${ }^{2}$ Electrical Engineering Department, Rongcheng College of Harbin University of Science and Technology, Weihai, P. R. China \\ ${ }^{3}$ Mechanical Engineering Department, Rongcheng College of Harbin University of Science and Technology, Weihai, P. R. China
}

Email address:

195453567@qq.com (Geng Liu), 17863123192@163.com (Zhen Li)

${ }^{*}$ Corresponding author

\section{To cite this article:}

Geng Liu, Xiao Han, Zhen Li. Urban Smart Growth Mathematical Model and Application. Applied and Computational Mathematics. Vol. 7, No. 3, 2018, pp. 83-88. doi: 10.11648/j.acm.20180703.12

Received: June 1, 2018; Accepted: June 22, 2018; Published: June 26, 2018

\begin{abstract}
In view of urban sprawl brought about by urbanization development, this paper establishes a weighted comprehensive evaluation model to measure the city's smart growth status. Bordeaux is selected as the research object, and relevant data are collected and processed. The data is then substituted into the established model to solve the problem. The results show that some indicators in the city are still at a poor level. Combining the indicators with higher weights and lower scores in the evaluation results, a better urban smart growth plan was proposed. Finally, the ARIMA forecasting model is used to predict the indicators in the future more than ten years. The results verify the effectiveness of the urban smart growth plan and the potential of the plans.
\end{abstract}

Keywords: Urban Smart Growth, Weighted Comprehensive Evaluation, ARIMA Forecast

\section{Introduction}

Smart growth is the urban planning and management theory proposed by the American planning community in the late 1990 s to cope with the disorderly spread of urban suburbanization and the resulting land resource and environmental problems [1]. The smart growth theory aims to build a city with economic prosperity, social equity, and environmentally sustainable development [2]. It integrates the use of other economic and social policies on the basis of urban spatial expansion theory [3], and plays an effective role in the urban growth management practices.

As a new idea of urban development, smart growth concept has gradually matured and made great progress in many countries. The smart growth concept emphasizes the comprehensive and coordinated development of the social economy and the resources and environment, and promotes the compact, centralized and efficient development of the city through the replacement of urban land use function, the delimit of the urban control boundary, the protection of the ecological environment and the old city transformation [4]. With the rapid development of urbanization and urban economy, cities are experiencing a historical process of rapid expansion of space scale and huge changes in spatial structure. The urban land scale is expanding rapidly, the land resources are destroyed by extensive urbanization, and the urban ecological environment and the quality of life of the residents are also adversely affected. Therefore, it is particularly important to choose a reasonable urban smart growth strategy to guide the healthy and orderly development of urbanization.

In view of the smart growth of cities, scholars have studied this issue. In the literature [5], the economy, society, environment and population of the city are used as the main indexes to measure the smart growth, and the differential equation model is set up, the dynamic changes of each index are predicted and analyzed, and the influence of each index on the smart growth of the city and the regulation effect on the sustainable development of the city in the future are studied. In the literature [6], based on the principal component analysis (PCA), the multiple impact factors that affect the realization of the smart growth of a city consist of three comprehensive indicators including economy, environment and energy. And 3E coordination degree theory is used to measure whether a city can achieve smart growth, so as to provide a reasonable basis for the smart growth of a 
city. The literature [7] constructs the urban smart growth evaluation index system according to the "three E principle", and uses the fuzzy comprehensive analysis and entropy method to set up a smart growth evaluation model for the selected index, and evaluates the designated city. The shrewd growth evaluation, index prediction and index potential evaluation were established by grey theory and Logistic regression, and the smart growth target of the city was predicted and evaluated. Based on the weighted comprehensive evaluation method and the ARIMA time series model, this paper analyzes the urban smart growth, and studies the impact of the indicators on the smart growth of the city and the regulation of the sustainable development of the city in the future.

\section{Research Foundation}

\subsection{Weighted Comprehensive Evaluation}

Weighting method is one of the common methods for solving multi-objective evaluation problems [8]. The basic idea of weighted evaluation method is to assign different weight coefficients to each of the selected indicators to calculate each goal. The significance of each target is the corresponding real number weights [9]. It is assumed that there are $n$ objects to be evaluated in an evaluation system, and there are $\mathrm{m}$ evaluation factors to form an evaluation index set. The weighted evaluation mathematical model is

$$
\begin{gathered}
\max F_{j}=\max \left\{F_{1}, \quad F_{2}, \quad \cdots, \quad F_{n}\right\} \\
F_{j}=\sum_{i=1}^{m} r_{i j} W_{i} \triangleq r_{j} W^{T} \leq 1 \\
\sum_{i=1}^{m} W_{i}=1 \\
W_{i}>0
\end{gathered}
$$

Where: represents the utility value of the $i$-th indicator of the $j$-th scheme, and represents the weight of the $i$-th indicator.

\subsection{Time Series Analysis}

ARIMA time series model is a high accuracy prediction model, the basic idea is that some time series are a set of random variates that depend on $t$ [10]. Although the single sequence value of the time series has is probabilistic, the change of the entire sequence has a certain regularity and can be approximated by the related mathematical model [11]. Proceed as follows:

Step 1: Take $n$ measures on a time series to get the sample $Z_{1}, Z_{2}, \cdots, Z_{n}$

Step 2: Data preprocessing: set $w_{t}=Z_{t}-\bar{Z}\left(\bar{Z}=\frac{1}{n} \sum_{i=1}^{n} Z_{i}\right)$, and get $n$ dates: $w_{1}, w_{2}, \cdots, w_{n}$
Step 3: Calculate the data: the sample self-covariance function $r_{k}$, the sample autocorrelation function $\rho_{k}$, the partial correlation function $\varphi_{k}$, among them: $k=0,1,2, \cdots, n$;

$$
\begin{gathered}
r_{k}=\frac{w_{1} w_{1+k}+w_{2} w_{2+k}+\cdots+w_{n-k} w_{n}}{n} \\
\rho_{k}=\frac{r_{k}}{r_{0}}
\end{gathered}
$$

Step 4: Stationary random process $\left\{Y_{t}\right\}$, satisfying the $p$ order stochastic difference equation and the following conditions:

$$
Y_{t}=\varphi_{1} Y_{t-1}+\cdots+\varphi_{p} Y_{t-p}+\alpha_{t}+\theta_{1} \alpha_{t-1}+\cdots+\theta_{q} \alpha_{t-q}
$$

With lag operator that:

$$
\begin{gathered}
\phi(L) Y_{t}=\Theta(L) \alpha_{t-q} \\
\phi(L)=1-\varphi_{1} L-\cdots-\varphi_{p} L^{p} \\
\Theta(L)=1-\theta_{1} L-\cdots-\theta_{q} L^{q}
\end{gathered}
$$

Among them: $L$ is lag operator; $\phi(L)$ is the $L$ of $p$ order autoregressive coefficient polynomial; $\Theta(L)$ is the $L$ of $q$ order moving average coefficient polynomial; $\varphi_{i}(i=1,2, \cdots, p)$ is autoregressive operator; $\theta_{j}(j=1,2, \cdots, q)$ is moving average operator; $\left\{\alpha_{t}\right\}$ is zero-mean white noise sequence.

\section{Model Establishment and Result Analysis}

\subsection{Weighted Comprehensive Evaluation Model}

Based on the principle of smart growth, four primary indicators of growth models and trends, protection of natural resources, environmental quality, and traffic quality were selected, as well as their corresponding secondary and tertiary indicators. In order to reflect the importance of different levels of indicators, we use the analytic hierarchy process to determine the weight of the indicators.

1. Construct a judgment matrix and assign value

Make a pairwise comparison for each element on the same level about the importance of a principle on the previous level, with one to nine scales, structure comparison matrix. In accordance with the guidelines of the importance of indicators for targets, indicators for each of the two criteria for a ratio, build judgment matrix $A_{1}, A_{2}$ and $A_{3}$.

For the four dimensions of urban growth models and trends, natural resource protection, environmental quality, and traffic quality, a judgment matrix such as formula (11) was established. 


$$
A_{1}=\left(\begin{array}{cccc}
1 & 2 & 2 & 3 \\
1 / 2 & 1 & 1 & 3 \\
1 / 2 & 1 & 1 & 3 \\
1 / 3 & 1 / 3 & 1 / 3 & 1
\end{array}\right)
$$

For the three indicators of scale and length, land use, and centralization, we have established a judgment matrix such as formula (12).

$$
A_{2}=\left(\begin{array}{ccc}
1 & 1 / 3 & 3 \\
3 & 1 & 7 \\
1 / 3 & 1 / 7 & 1
\end{array}\right)
$$

For the three indicators of park green space, air environment, and acoustic environment, we established a judgment matrix such as formula (13).

$$
A_{3}=\left(\begin{array}{ccc}
1 & 3 & 5 \\
1 / 3 & 1 & 3 \\
1 / 5 & 1 / 3 & 1
\end{array}\right)
$$

For other selected indicators, we believe that the impact on smart growth is significant and the weights are evenly divided.

2. Calculate weight vector and consistency check

In order to determine the allowable range of the inconsistency of the judgment matrix, a consistency check is used to calculate the weight vector. The relative weights of the elements being compared for the criterion are calculated by the judgment matrix, and the consistency check is performed to define the consistency index as formula (14).

$$
C I=\frac{\lambda-n}{n-1}
$$

The greater the $C I$, the more inconsistent. To measure the size of the $C I$, introduce a random consistency index $R I$, calculate the $C I$ to get $R I$, and define the consistency ratio $C R$ as in formula (15).

$$
C R=\frac{C I}{R I}
$$

Among them: when $C R<0.1$, pass the consistency test. If passed, the eigenvector corresponding to the largest characteristic root serves as a weight vector.

Based on the above analysis, the $C R$ of $A_{1}, A_{2}$, and $A_{3}$ are shown in Table 1.

Table 1. CR value of judgment matrix.

\begin{tabular}{ll}
\hline Judgment matrix & $\boldsymbol{C R}$ value \\
\hline$A_{1}$ & 0.0227 \\
$A_{2}$ & 0.0370 \\
$A_{3}$ & 0.0046 \\
\hline
\end{tabular}

Because $C R<0.1$, so through the consistency test, and find the eigenvector of the largest eigenvalue of the judgment matrix at this time is the basic weight vector, the result is as the formula (16) (17) (18).

$$
\begin{aligned}
& W_{1}=\left\{\begin{array}{llll}
0.4146 & 0.2436 & 0.236 & 0.0982
\end{array}\right\} \\
& W_{2}=\left\{\begin{array}{lll}
0.6370 & 0.2583 & 0.1047
\end{array}\right\} \\
& W_{3}=\left\{\begin{array}{lll}
0.2426 & 0.6694 & 0.879
\end{array}\right\}
\end{aligned}
$$

Therefore, we can calculate the weights among the indicators. The results are shown in Table 2- Table 4.

Table 2. Dimension's indicators weight.

\begin{tabular}{ll}
\hline Dimension's indicators & \\
\hline Index & Weights \\
\hline Growth Models and Trends & 0.4789 \\
Natural resources protection & 0.2383 \\
Environmental Quality & 0.0838 \\
Traffic quality & 0.1990 \\
\hline
\end{tabular}

Table 3. Secondary indicators weight.

\begin{tabular}{ll}
\hline Secondary indicators & \\
\hline Index & Weights \\
\hline Scale and length & 0.6370 \\
Land use & 0.2583 \\
Centralized & 0.1047 \\
Policy development & 0.2000 \\
Policy effectiveness & 0.8000 \\
Parkland & 0.2426 \\
Air environment & 0.6694 \\
Acoustic environment & 0.0879 \\
Land traffic & 0.5000 \\
Land traffic & 0.5000 \\
\hline
\end{tabular}

Table 4. Third-level weight.

\begin{tabular}{ll}
\hline Index & Weights \\
\hline Urban population development trend & 0.333 \\
Urban Employment Development Trend & 0.333 \\
Economic trends & 0.333 \\
Unutilized land ratio & 0.500 \\
Change in land use ratio & 0.500 \\
Centralization & 1.000 \\
Reasonable policy & 1.000 \\
Changes in the number of agricultural land & 0.333 \\
Agricultural Structure Change Trend & 0.333 \\
Utilization of natural resources & 0.333 \\
Per capita park green area & 0.500 \\
Built-up area greening rate & 0.500 \\
Air quality is not good & 1.000 \\
Road traffic noise average equivalent sound level & 0.500 \\
Urban area noise average equivalent sound level & 0.500 \\
Per capita road length & 0.333 \\
Number of cars & 0.333 \\
Bus line length & 0.333 \\
Number of ships & 0.500 \\
Number of aircraft & 0.500 \\
\hline
\end{tabular}

\section{Multi-index weighted evaluation}

After determining the weights of the indicators, a multi-index weighted evaluation method is used to evaluate the objects to be evaluated and give corresponding scores. Construct a set of evaluation grades and specify that the score be between $(0,1), 0$ to 0.2 is very poor, 0.2 to 0.4 is bad, 0.4 to 0.6 is fair, 0.6 to 0.8 is good, 0.8 -Excellent between -1.0 
points. After the data is subjected to the same orientation and non-dimensionalization, weighted synthesis method is used to weight the individual evaluation values to obtain the total score of the evaluation.

\section{Case analysis solution}

We chose Bordeaux, a developed country city located on the coast, to collect data on the city's indicators. Due to the different nature of the collected data, some are positive data and some negative data. Therefore, we need to carry out the same direction of data processing, so that all data from the same point of view to explain the overall. At the same time, due to the different data sizes of different indicators, the indicators should be dimensionlessly processed to calculate their individual evaluation values. In addition, the Min-Max normalization and Arctan function transformation methods were used to correct the maximum and minimum values in the data. The single evaluation values of Bordeaux's indicators were calculated as shown in Table 5.

Table 5. The evaluation value of Bordeaux indicators.

\begin{tabular}{ll}
\hline Index & Score \\
\hline Urban population development trend & 0.718 \\
Urban Employment Development Trend & 0.707 \\
Economic trends & 0.717 \\
Land use ratio & 0.329 \\
Change in land use ratio & 0.732 \\
Centralization & 0.729 \\
Reasonable policy & 0.741 \\
Change in the number of agricultural land & 0.720 \\
Changes in the structure of agricultural land & 0.718 \\
The exploitation of natural resources & 0.679 \\
Per capita park green area & 0.867 \\
Built-up area greening rate & 0.9894 \\
Good air quality & 0.588 \\
Road traffic noise average equivalent sound level & 0.018 \\
Urban area noise average equivalent sound level & 0.976 \\
Per capita road length & 0.870 \\
Number of cars & 0.766 \\
Bus line length & 0.849 \\
Number of ships & 0.708 \\
Number of aircraft & 0.707 \\
\hline
\end{tabular}

After data processing, we will substitute various indicators into the evaluation system of smart growth for evaluation and analysis. The final score of smart growth evaluation was obtained by weighted comprehensive calculation. The score of smart growth of Bordeaux in France was 0.6987, which was in a good level. Judging from the individual evaluation values of various indicators, most of them are above 0.6 (good), and from the overall point of view of the overall rating, the results of urban indicators have also reached a good level. This shows that the city's current growth plan is in line with the principle of smart growth, and the city's smart growth status is at a good level. However, some individual evaluation indicators are at the general level of 0.4-0.6, and some single evaluation indicators are even at a relatively low level of $0.2-0.4$. Therefore, in order to obtain better evaluation scores for smart growth, a more reasonable and smart growth plan should be proposed.

\subsection{Smart Growth Plan}

In order to propose a more reasonable smart growth plan, consideration should be given to the weight of each indicator and the individual evaluation value of each indicator. Indicators with higher weights and lower individual scores should be given priority. Therefore, we combine the weights of individual indicators and the overall situation of individual evaluation values to rank. The results of comprehensive ranking of indicators are shown in Table 6. According to the comprehensive ranking of the indicators, we can reasonably select indicators that should focus on development.

Table 6. Overall Ranking of Bordeaux Indicators.

\begin{tabular}{ll}
\hline Index & Rank \\
\hline Unutilized land ratio & 1 \\
Exploitation of natural resources & 2 \\
Urban Employment Trends & 3 \\
Good air quality rate & 4 \\
Economic trends & 5 \\
Urban Population Trend & 6 \\
Road traffic noise average equivalent sound level & 7 \\
Number of aircraft & 8 \\
Changes in the structure of agricultural land & 9 \\
Changes in the number of agricultural land & 10 \\
Number of ships & 11 \\
Change in land use ratio & 12 \\
Concentration & 13 \\
Policy rationality & 14 \\
Number of cars & 15 \\
Bus line length & 16 \\
Per capita park green area & 17 \\
Average length of road & 18 \\
Built-up area greening rate & 19 \\
Urban area noise average equivalent sound level & 20 \\
\hline
\end{tabular}

From the table, we can see that there are major problems in land use, natural resources, and urban employment in Bordeaux, France, and there is a need for improvement in terms of urban population, economic growth, transportation quality, and environmental quality. In combination with Bordeaux's own economic, geographical and demographic factors, we propose the following growth plan under the framework of three goals and ten smart growth principles.

(1) Rationally use land resources to increase land utilization and avoid blind expansion.

(2) Save natural resources, protect the environment, and improve people's living standards.

(3) Continue to develop the wine industry, improve the export of high-quality wines, improve trade in trade development, and increase employment opportunities.

\subsection{Plan Efficiency Evaluation}

In order to quantify the impact of the smart growth plan, we should change the values of the corresponding indicators of the plan, and then use the previous smart growth evaluation system to evaluate the improved indicators. For the change of the indicator value, it cannot violate the objective development law. Therefore, we use the ARIMA forecasting model, based on the forecast value of the 
indicator after 10 years, put forward the expected target value of the indicator, and evaluate the benefit of the smart growth plan we have established.

According to our smart growth plan, we selected eight relevant indicators, namely land unavailability, natural resources, employment, air quality, economic trends, population trends, changes in the structure of agricultural

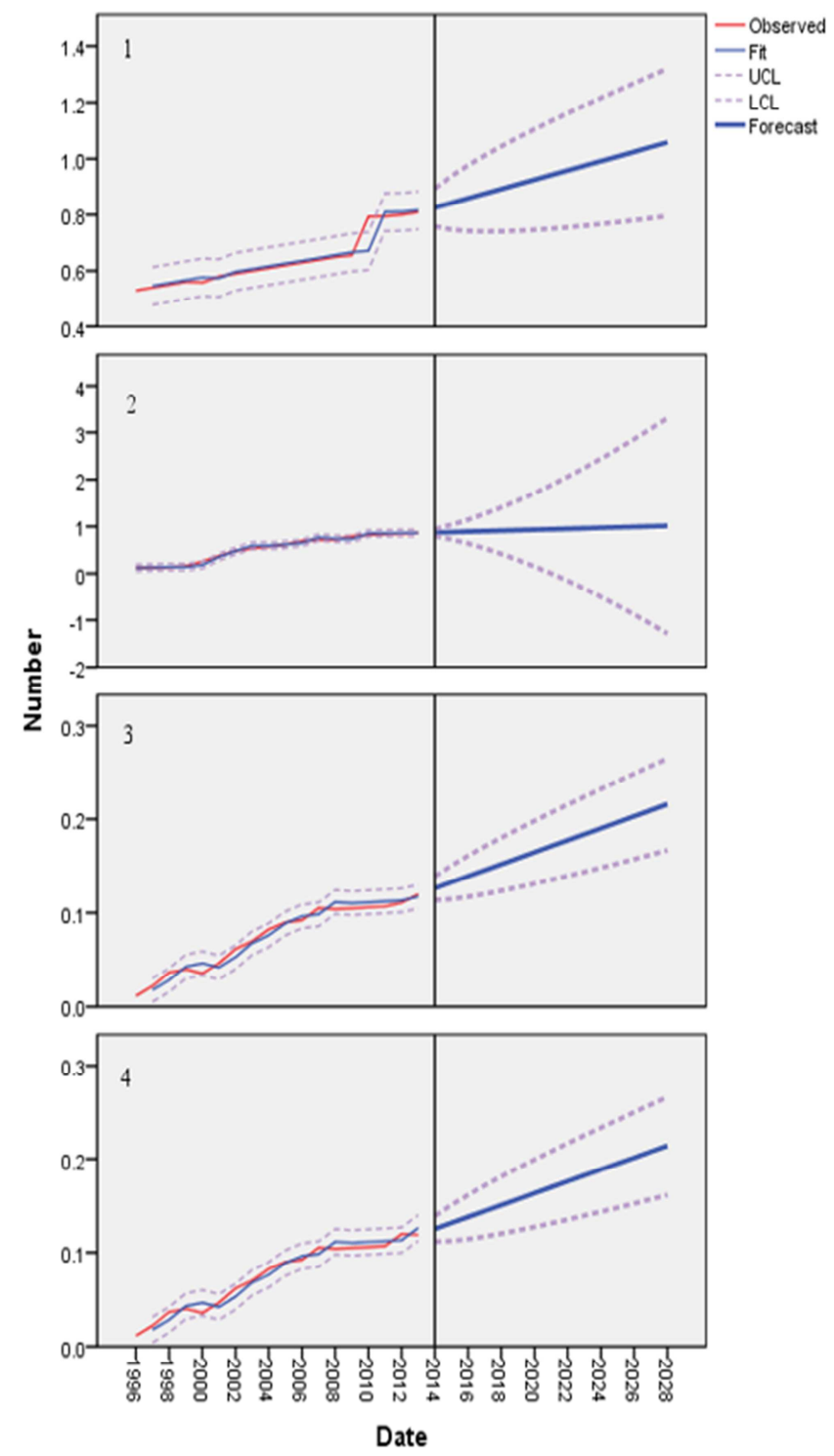

land and road traffic noise equivalent levels.

In combination with the established ARIMA forecasting model, through the previous data, the future changes in the index values for more than ten years are predicted and analyzed, and on this basis, the expected target values of important indicators in the plan are reasonably specified. The forecast results are shown in Figure 1.
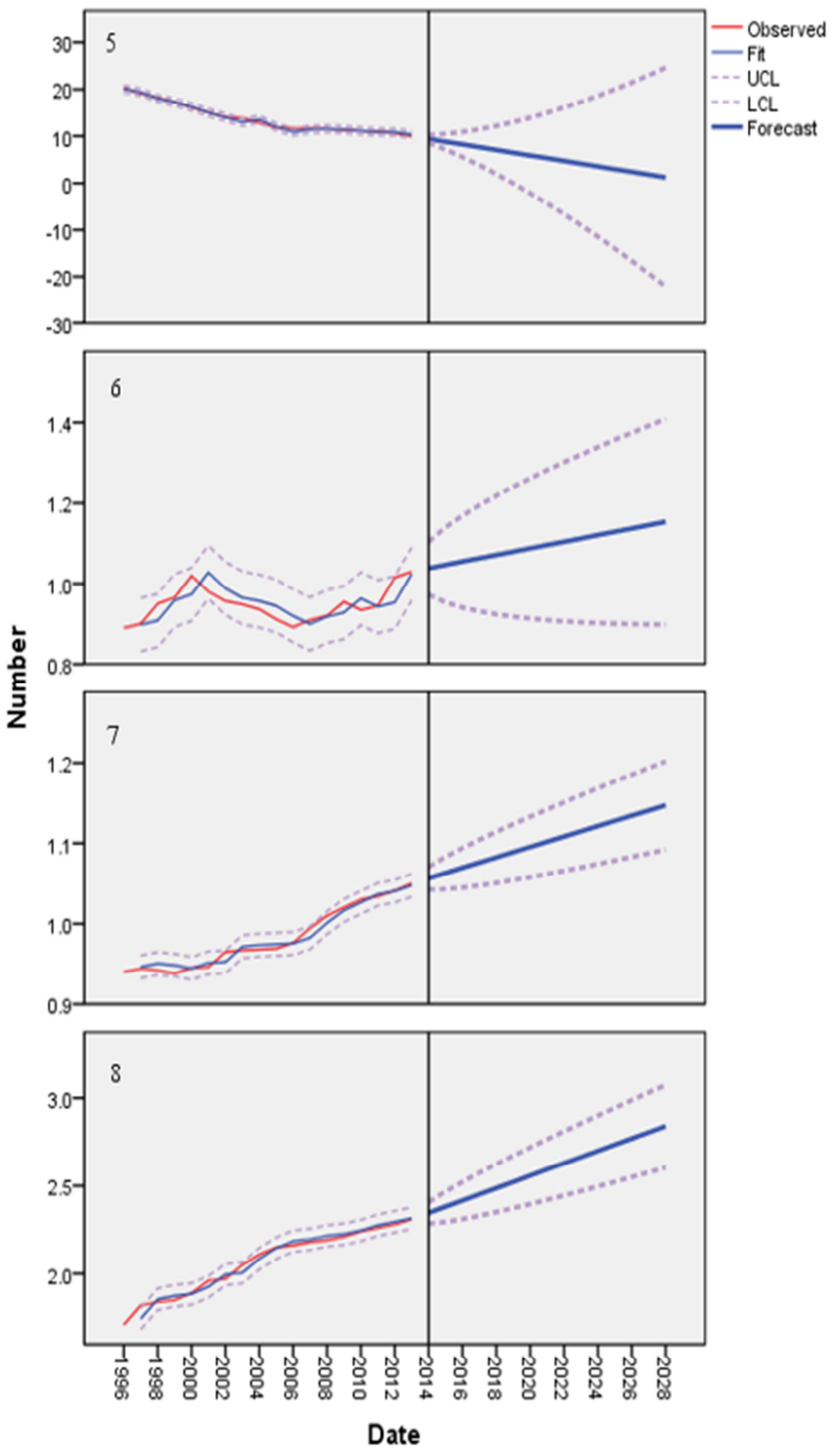

Figure 1. Forecast result of each index.

Where: the figures 1-8 are shown as: 1: The rate of unused land, 2: Natural resource exploitation rate, 3: Employment rate, 4: Air quality, 5: Trend of economic change, 6: Population development rate, 7: Structure change of agricultural land, 8: Road noise level

According to the results of the forecast and the proposed plan, the expected target value for the next five years will be set as the forecast value of the indicator after 10 years, and a savvy growth plan will be quantified. Through the rational quantification of the plan, we can quantify the scores of city smart growth after the implementation of the plan. Based on the predicted value of the indicator after 10 years, the expected target value of the indicator can be proposed and a new single evaluation value can be obtained as shown in
Table 7.

Table 7. Bordeaux improved evaluation value.

\begin{tabular}{ll}
\hline Index & Score \\
\hline Urban population development trend & 0.717 \\
Urban Employment Development Trend & 0.709 \\
Economic trends & 0.720 \\
Land use ratio & 0.450 \\
Change in land use ratio & 0.739 \\
Centralization & 0.729 \\
\hline
\end{tabular}




\begin{tabular}{ll}
\hline Index & Score \\
\hline Reasonable policy & 0.741 \\
Change in the number of agricultural land & 0.722 \\
Changes in the structure of agricultural land & 0.722 \\
The exploitation of natural resources & 0.705 \\
Per capita park green area & 0.867 \\
Built-up area greening rate & 0.974 \\
Good air quality & 0.657 \\
Road traffic noise average equivalent sound level & 0.989 \\
Urban area noise average equivalent sound level & 0.976 \\
Per capita road length & 0.870 \\
Number of cars & 0.768 \\
Bus line length & 0.849 \\
Number of ships & 0.708 \\
Number of aircraft & 0.707 \\
\hline
\end{tabular}

It can be concluded from Table 7 that most of the individual evaluation values have reached good levels after the implementation of the formulated plan, and the lowest indicators have also reached the general level. After the plan, Bordeaux's comprehensive savvy growth evaluation score is expected to reach 0.7165 . The level of growth has significantly improved.

\subsection{Plan Potential Evaluation}

The savvy growth plan we have developed is based on the comprehensive rankings of various indicators. When evaluating the potential of each plan, we should change the corresponding indicator values of each plan by controlling variables, and the obtained indicators should be improved. Through the evaluation system established before, the evaluation scores for smart growth after the implementation of a single plan are shown in Table 8 , and the results are used to define the potential of the plan.

Table 8. Smart growth rating after implementation of each program.

\begin{tabular}{llll}
\hline \multirow{2}{*}{ Initial samrt growth score } & Plan A & Plan B & Plan C \\
\cline { 2 - 4 } & Separate implementation & Separate implementation & Separate implementation \\
\hline 0.6987 & 0.7118 & 0.7050 & 0.7061 \\
\hline
\end{tabular}

The table shows that Bordeaux's planned potential ranking should be:

(1) Make rational use of land resources to increase land utilization and avoid blind expansion;

(2) Continue to develop the wine industry, improve the export of high-quality wines, improve trade in transport development, and increase employment opportunities;

(3) Save natural resources, protect the environment and improve people's living standards.

\section{Conclusion}

In order to solve the problem of urban sprawl and promote urban smart growth, this paper establishes a weighted comprehensive evaluation model, combines quantitative analysis with qualitative analysis, and converts multi-level, multi-target, and difficult-to-quantify complex problems into multi-level single-target problems. According to the evaluation results, the problems existing in urban development are pointed out, and then according to the unique economic model and cultural background of the city, corresponding smart growth plans are proposed. In order to verify the effectiveness of the plan, an ARIMA forecasting model was established, and the predicted values of the selected indicators after more than a decade were obtained. The prediction results proved the rationality of the smart growth plan. However, when using the analytic hierarchy process, a judgment matrix is obtained based on expert experience, and the prediction result is subjective, easily causing some errors, and needs improvement.

\section{References}

[1] Cheng Maoji. Research on Nanjing City Growth Evaluation and Optimization Based on Smart Growth [D]. Nanjing Normal University, 2012.
[2] Ruan Zhanfu. Research on Urban Spatial Expansion Based on Smart Growth [D]. Northwest Normal University, 2009.

[3] Guan Jing. A Review of Research on Smart Growth [J]. Research on Financial and Economic Issues, 2013 (02): 26-31.

[4] Ruan Zhanfu. Research on Urban Spatial Expansion Based on Smart Growth [D]. Northwest Normal University, 2009.

[5] Zhou Rong, Wang Qian, Wang Yanhui. Mathematical model of "smart growth" in cities [J]. Mathematic Modeling and Applications, 2017, 6 (02): 16-24.

[6] Li Qin. Evaluation of Urban Smart Growth Based on Principal Component Analysis [J]. Urban Construction Theory Research, 2016 (23): 11.

[7] Liu Yan, Zhu Jiaming. Evaluation and Grey Prediction of Urban Smart Growth Based on Fuzzy Comprehensive Method [J]. Journal of Chifeng College (Natural Science Edition), 2017, 33 (15): 29-32.

[8] Wang Bangli, Huang Wenquan, Li Kaishi. Research on Product Material Selection Based on Analytic Hierarchy Process and Weighted Evaluation Method [J]. Journal of Sichuan University of Science \& Engineering (Natural Science Edition), 2013, 26 (05): 71-73.

[9] Sun Huichao, Cheng Gang, Li Yuli, Huang Ningning. Study on Comprehensive Evaluation System of Urbanization Development Level [J]. Journal of Henan University of Urban Construction, 2016, 25 (05): 53-60+87.

[10] Li Hailin. Research on feature representation and similarity measurement methods in time series data mining [D]. Dalian University of Technology, 2012.

[11] Yuan Jidong, Wang Zhihai. A Summary of Time Series Representation and Classification Algorithms [J]. Computer Science, 2015, 42 (03): 1-7. 